\title{
Diagnostic accuracy of contrast-enhanced digital mammography in comparison with sonomammography for characterization of focal asymmetries
}

Gelan Ali Mahmoud Soliman ${ }^{1 *}$, Shaimaa Abdelsattar Mohammad ${ }^{1}$, Mohamed El-Shinawi ${ }^{2}$ and Nermeen Nasry Keriakos ${ }^{1}$

\begin{abstract}
Background: Mammographic focal asymmetry represents normal breast tissue, benign, or malignant lesions. Accurate characterization is important for better management. The study evaluates diagnostic accuracy of contrastenhanced digital mammography (CEDM) for characterization of focal asymmetries seen in 2D mammography.

Results: The study was done prospectively on 38 females among 360 patients who underwent baseline sonomammographic assessment for diagnostic and screening purposes. Complementary ultrasound was performed only when a finding was detected in cases of screening mammograms. Focal asymmetries were evaluated according to Breast Imaging Reporting and Data System (BI-RADS) lexicon 2013. CEDM was performed and followed by ultrasound (US) guided core biopsy for solid lesions or aspiration for cystic lesions. CEDM processing resulted in recombined image showing enhancing abnormality. Low energy image and recombined image findings were analyzed blindly and classified into focus enhancement, mass enhancement, non-mass enhancement, and non-enhanced lesions. CEDM and sonomammography findings were compared regarding pathological probability and multiplicity. Histopathology was the reference standard.

Mass enhancement showed strong correlation with malignant pathology. Non-mass enhancement showed no correlation with particular pathology. All non-enhanced focal asymmetries were benign in pathology or normal tissue. Rim enhancement needed second look ultrasound evaluation. CEDM was superior to sonomammography with higher sensitivity $(77.8 \%, 65.7 \%$ respectively), NPV $(0.8,0.6)$, accuracy $(0.6,0.2)$ but lower specificity $(81.8 \%$ vs. $100 \%$ ). Multiplicity detection by CEDM was $26.3 \%$ and by sonomammography was $10.5 \%$.

Conclusion: CEDM is more accurate than sonomammography in determination of normal tissue, benign, or malignant lesions in cases of mammographic focal asymmetry. CEDM is more accurate in detection of multiplicity. Undesired biopsies were avoidable with proper management of suspicious and malignant lesions.
\end{abstract}

Keywords: Focal asymmetry, Contrast, Mammography

\footnotetext{
* Correspondence: gigisoliman79@yahoo.com

1 Radiodiagnosis Department, Ain Shams University, Ramsees Street, Al

Abasia, Cairo 11517, Egypt

Full list of author information is available at the end of the article
}

\section{Springer Open}

(c) The Author(s). 2020 Open Access This article is licensed under a Creative Commons Attribution 4.0 International License, which permits use, sharing, adaptation, distribution and reproduction in any medium or format, as long as you give appropriate credit to the original author(s) and the source, provide a link to the Creative Commons licence, and indicate if changes were made. The images or other third party material in this article are included in the article's Creative Commons licence, unless indicated otherwise in a credit line to the material. If material is not included in the article's Creative Commons licence and your intended use is not permitted by statutory regulation or exceeds the permitted use, you will need to obtain permission directly from the copyright holder. To view a copy of this licence, visit http://creativecommons.org/licenses/by/4.0/. 


\section{Background}

Mammography is the cornerstone screening tool for breast cancer. The earliest sign of breast cancer can be depicted on a mammogram [1]. Focal asymmetry is a mammographic asymmetric density seen on two projections, hence, a real finding rather than superimposition [2]. It may represent normal tissue, benign or malignant lesions, or architectural distortion [3].

CEDM provides morphologic and functional information enabling distinguishing malignant from benign lesions [4]. CEDM provides immediately available, clinically useful information that change the diagnostic and treatment strategy. CEDM improves sensitivity, specificity, and accuracy for breast cancer detection and staging [5].

There are many reports investigating the value of CEDM in the assessment of breast lesions [3, 6, 7]. However, few papers assessed its value in cases of mammographic focal asymmetry in particular to verify the pathological nature of those lesions [8].

The aim of the study is to evaluate diagnostic accuracy of CEDM for characterization of mammographic focal asymmetries.

\section{Methods}

After the institutional ethical committee approval, a prospective study was performed between January and March 2019. The procedure was explained for each patient and written informed consent was signed. The study was performed at the breast radiology clinic including patients presented to the breast surgery clinic complaining of palpable mass, nipple discharge, or mastalgia or seeking screening. They underwent full history and clinical evaluation. Among 360 patients who underwent baseline sonomammographic assessment for diagnostic and screening purposes, only 38 female patients proved to have focal asymmetry. Their age ranged between 26 and 72 years old. Following the American Society of Breast Surgeons, a mammogram was routinely done when the patient was 35 years or above [9]. In screening cases, complementary ultrasound was performed only when the finding of focal asymmetry was detected and hence included in our study. We had only three cases below 35 years who complained of palpable breast masses and hence were initially assessed by US, followed by CEDM. One case out of three was diagnosed by biopsy as malignant and was scheduled for CEDM to assess multiplicity; the other 2 cases had positive family history and palpable findings and planned for CEDM where focal asymmetry was detected in the low energy images.

\section{Procedure details}

After complete menstrual, medical, and family history taking, ultrasound and 2D mammography were performed by using ultrasonic machine (Samsung Accuvix XG) and full-field digital mammographic machine (GE Pristina). At least 2 mammographic views are usually acquired mainly craniocaudal and mediolateral oblique views. Before contrast administration, serum creatinine was checked with calculation of estimated GFR. Patients were asked to be fasting 6-8 $\mathrm{h}$ before the examination. Neck apron was applied and a wide bore cannula was inserted in the arm. Iodinated contrast material was injected.

CEDM as a dual-energy technique was applied. Lowand high-energy images were obtained after contrast injection and subtraction of pre-contrast from the postcontrast images [10].

Technique started by contrast injection followed by compression of the appropriate breast into the desired projection. Acquisitions were performed at 2, 4, 6, and 8 min with breath holding. Additional projections can be acquired at $10 \mathrm{~min}$. Each acquisition was formed of high and low energy images $20 \mathrm{~s}$ in between. Breast can be uncompressed between acquisitions. Visibility of lesions decreases by time due to wash out. The background in

Table 1 Data representing CEDM, pathological, and sonomammographic (BIRADS) findings

\begin{tabular}{|c|c|c|c|c|c|c|c|c|c|c|}
\hline & \multirow[t]{2}{*}{ No. } & \multicolumn{5}{|c|}{ Pathological diagnosis } & \multicolumn{4}{|c|}{ BIRADS } \\
\hline & & & ical types of benign lesions (no.) & Patk & s of malignant lesions (no.) & $\mathbf{a}$ & 1 & 3 & 4-5 & 6 \\
\hline \multirow[t]{4}{*}{ Enhancing mass } & 24 & 3 & Fibroadenoma (2) & 21 & IDC (18) & & & & 22 & 2 \\
\hline & & & & & $\mathrm{IDC}+\operatorname{ILC}(1)$ & & & & & \\
\hline & & & & & $\mathrm{IDC}+\mathrm{DCIS}(1)$ & & & & & \\
\hline & & & Abscess (1) & & $\operatorname{ILC}(1)$ & & & & & \\
\hline \multirow[t]{2}{*}{ Non-mass enhancement } & 8 & 6 & Abscesses (3) & 2 & 2 IDC & & & 1 & 7 & 0 \\
\hline & & & Granulomatous mastitis (3) & & & & & & & \\
\hline Enhancing focus & 1 & 1 & Abscesses (1) & & & & & 0 & 1 & 0 \\
\hline \multirow[t]{2}{*}{ No enhancement } & 5 & 3 & $\mathrm{EDH}(1)$ & & & 2 & 2 & 0 & 3 & 0 \\
\hline & & & Fibroadenoma (2) & & & & & & & \\
\hline
\end{tabular}

IDC invasive duct carcinoma, ILC invasive lobular carcinoma, DCIS ductal carcinoma in situ, EDH epithelial ductal hyperplasia

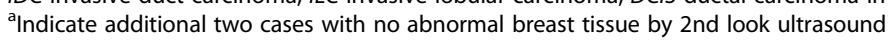


Table 2 CEDM, sonomammography, and pathology frequencies and percent

\begin{tabular}{llll}
\hline & $\begin{array}{l}\text { CEDM } \\
\text { Number (\%) }\end{array}$ & $\begin{array}{l}\text { Sonomammography } \\
\text { Number (\%) }\end{array}$ & $\begin{array}{l}\text { Pathology } \\
\text { Number (\%) }\end{array}$ \\
\hline Benign & $7(18.4 \%)$ & $1(2.6 \%)$ & $13(34.2 \%)$ \\
Malignant & $29(76.3 \%)$ & $36(92.1 \%)$ & $23(60.5 \%)$ \\
Normal & $2(5.2 \%)$ & $25(5.2 \%)$ & $2(5.2 \%)$ \\
Total & $38(100 \%)$ & $38(100 \%)$ & $38(100 \%)$ \\
\hline
\end{tabular}

low-energy image is subtracted and the high energy of contrast persists, allowing visibility of any enhancing abnormality [11].

In our study, images were obtained by modified fullfield digital mammography equipment. Contrast media, Omnipaque $300 \mathrm{ml}(1.5 \mathrm{~mL} / \mathrm{kg})$ was administered intravenously and manually followed by saline bolus. The breast was compressed properly and patient was asked to stop breathing. Decompression between exposures was allowed. Acquisition of the low energy image (26-32 $\mathrm{kVp})$ and high energy image (45-49 $\mathrm{kVp}$ ) was performed after 2 min from starting of injection in the following order $\mathrm{CC}$ view of the diseased side $(2 \mathrm{~min}), \mathrm{CC}$ of the normal side $(4 \mathrm{~min}), \mathrm{MLO}$ view of the diseased side (6 min) then MLO of contralateral side ( $8 \mathrm{~min})$. Image of the diseased side could be taken at $10 \mathrm{~min}$ if needed. Low energy images were evaluated for soft tissue and calcification details and recombined images were evaluated for any abnormal enhancement. Reading of low energy before and after CEDM was done by two different readers to avoid bias.

\section{Data analysis, reporting, and interpretation}

Sonomammographic and CEDM analysis were performed independently by two authors who were blinded to the final pathological diagnosis. Sonomammographic findings were classified according to BI-RADS, while the recombined energy image of CEDM was analyzed according to MRI lexicon and classified into focal enhancing lesion, mass enhancing lesions and non-mass enhancing lesions, and non-enhancing lesions.

Benign descriptors of mass enhancing lesions are circumscribed margin, regular shape, homogenous pattern, and faint enhancement. Suspicious descriptors are irregular shape, irregular or speculated margin, heterogeneous pattern, and moderate or intense enhancement. The most suspicious descriptors of mass enhancing lesions are irregular or speculated margin and irregular
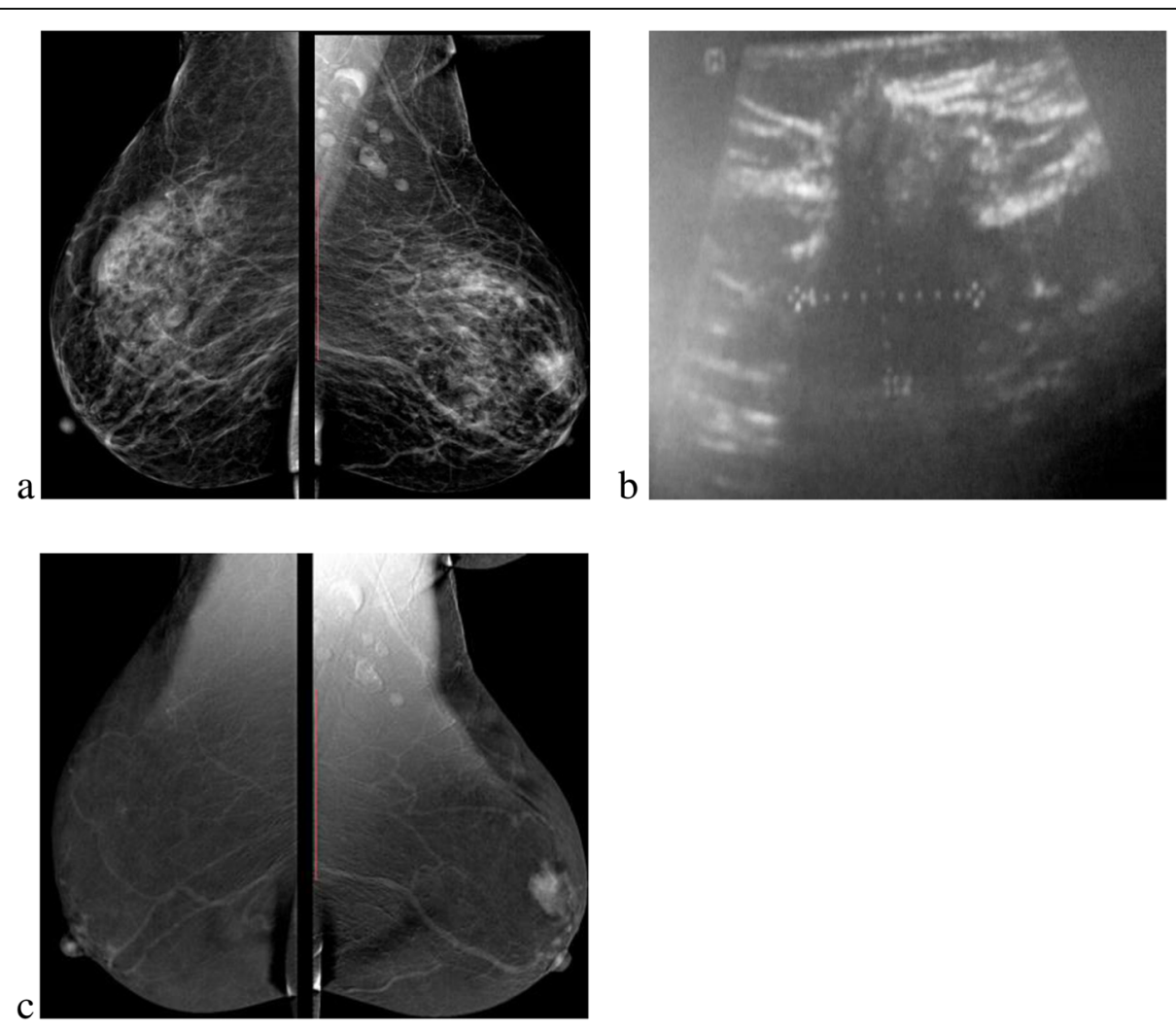

Fig. 1 A case of invasive ductal carcinoma grade II. Image (a) shows left focal asymmetry by low energy, (b) shows an irregular speculated margin hypoechoic lesion on ultrasound. c Recombined image of CEDM showing enhancing mass lesion of irregular shape, irregular margin, and intense enhancement 

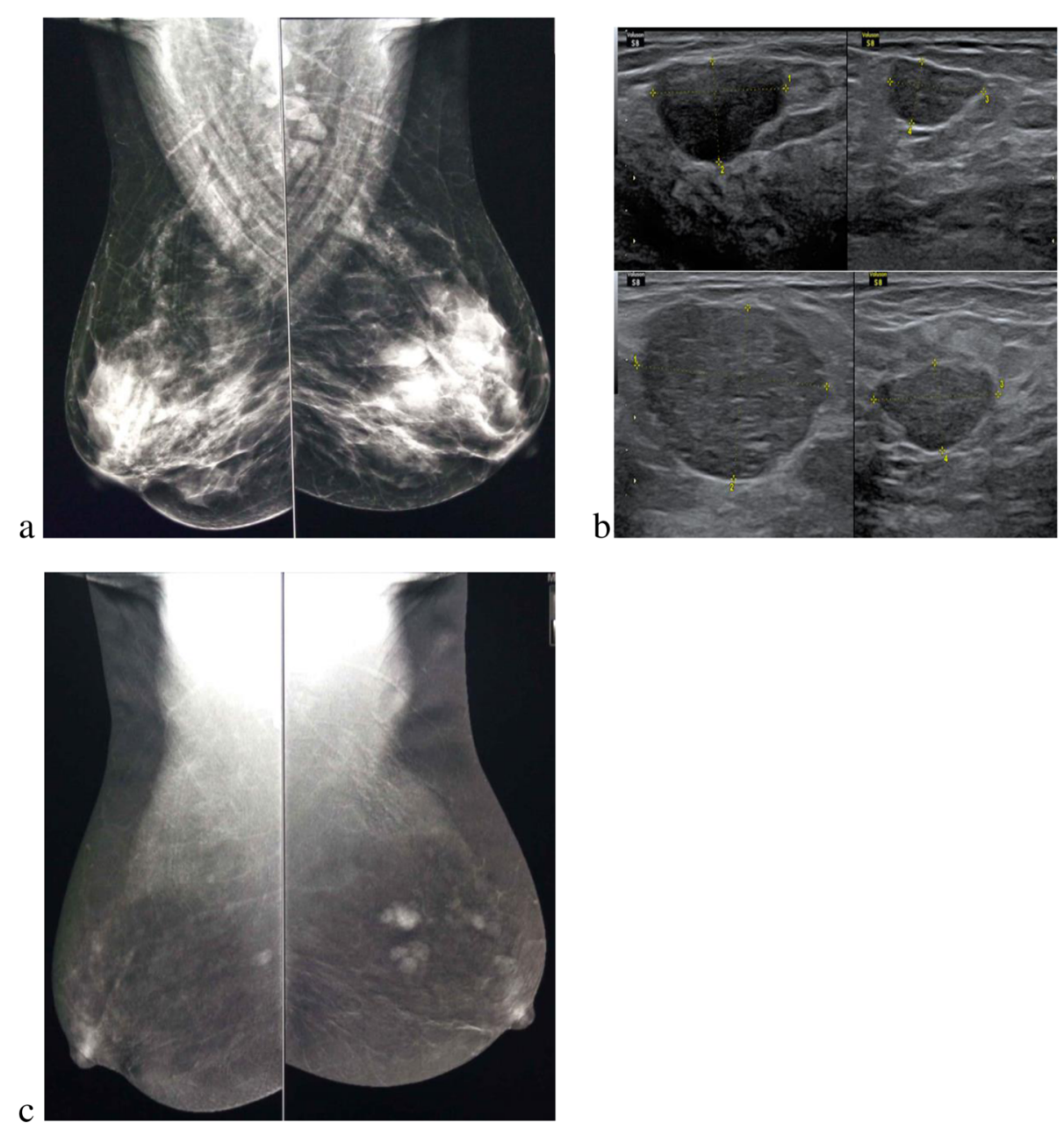

Fig. 2 A case of left multiple fibroadenomas presented in (a) as left upper outer and inner quadrants focal asymmetries in low energy image. b Ultrasound images of regular smoothly marginated hypoechoic fibroadenomas. c Recombined image of CEDM showing multiple mass enhancing lesions with internal non enhanced septae

shape. Benign descriptors of non-mass enhancing lesions are symmetrical, multi-regional, or diffuse disruption and homogenous enhancement. Suspicious descriptors are asymmetry, focal, ductal, segmental or regional distribution, and heterogeneous or clumped enhancement. The most suspicious descriptors of non-mass enhancing lesions are ductal, segmental, and focal distributions. Also, regional distribution with heterogeneous pattern is considered intermediately suspicious. Rim enhancement is an equivocal enhancement. As regard enhanced focus, the most suspicious descriptor for enhancing focus is the intensity of enhancement rather than multiplicity. Lesion multiplicity by sonomammography and CEDM was documented.

CEDM was followed by ultrasound-guided core biopsy for solid lesions or aspiration for cystic lesions. Second look US and reviewing low energy images were mandatory in suspicious enhancing lesions or rim enhancement. In cases of undetectable lesions by neither
CEDM nor second look US, screening follow-up was done every 6 months for 2 years for high-risk patients.

Sonomammographic and CEDM findings were compared to pathological results. Multiplicity in sonomammography and CEDM also was compared.

Data was processed statistically using IBM SPSS Statistics 21 and presented in tables and figures.

\section{Results}

\section{Sonographic findings}

Findings were classified according to BI-RADS as shown in Table 1.

BI-RADS probabilities revealed malignancy, benignity, and normal findings as in Table 2.

\section{CEDM findings}

1 Enhancing mass was the most common pattern. It was seen in 24 patients (63.1\%) (Figs. 1, 2, 3, 4). 

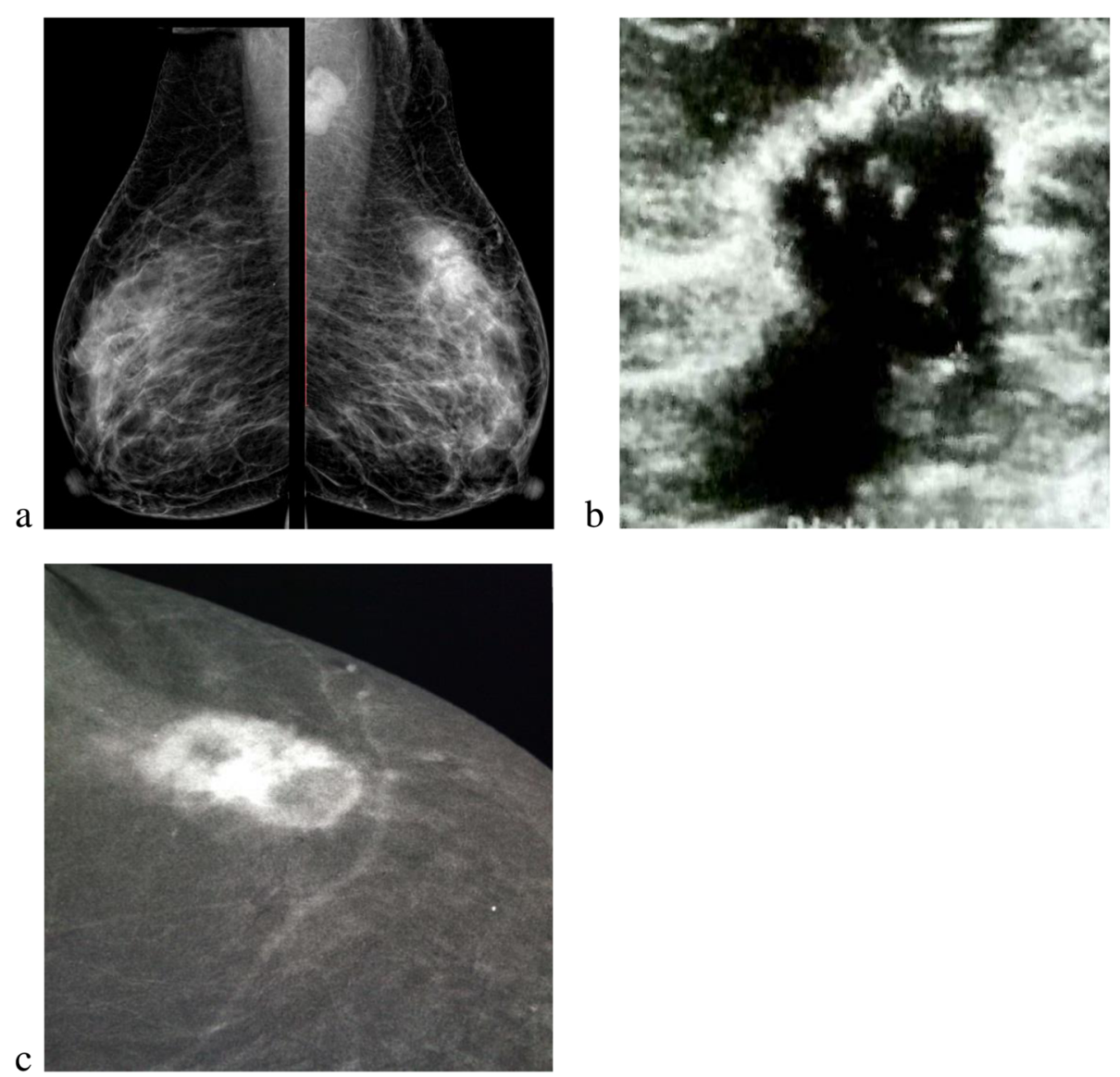

Fig. 3 A case of invasive ductal carcinoma represented by left focal asymmetry (a) and shows an irregular speculated margin hypoechoic mass on ultrasound (b). Recombined image of CEDM shows a mass with ring enhancement in the recombined image (c)

CEDM lesion descriptors showed marked predilection to malignant features. Irregular margin was seen in 22 patients (91.7\%) who were diagnosed pathologically as 21 malignant and 1 benign. The irregular shape and moderate and intense enhancement were seen in 21 patients (87.5\%) classified pathologically into 19 malignant and 2 benign. Heterogeneous enhancement was noticed in 14 patients (58.3\%). All of them categorized as true or suspicious malignant lesions by sonomammography and proved as malignant (Table 1).

2 Non-mass enhancement (Fig. 5) was detected in 8 patients (21\%). According to CEDM descriptors, non-mass enhancing lesions showed predilection to malignant features. Seven patients revealed suspicious distribution (ductal, segmental, and regional). They were diagnosed pathologically as 1 malignant and 6 benign cases. In contrast, only one case showed benign distribution (multiregional) and proved as malignant. As regard pattern of enhancement, 5 out of 8 cases $(62.5 \%)$ showed moderate or intense enhancement and proved pathologically as 2 malignant and 3 benign cases. All non-mass enhancing lesions showed heterogeneous enhancement. Sonomammography showed predominant suspicious malignant lesions in contrast to predominant benign histopathology (Table 1).

3 Enhancing foci were detected in only one case. It represented a benign inflammatory condition; foci were of irregular shape, irregular margin, and faint enhancement. Associated small abscesses appeared as rim enhancements. Sonomammography and pathological diagnosis are seen in Table 1.

4 Non-enhancement was detected in 5 cases (13\%). Three cases were BIRADS 4 and proved to be benign by histopathology. The rest 2 non-enhanced cases were BI-RADS 1 by US (Table 1) (Fig. 6).

Collectively, CEDM probabilities revealed malignancy, benignity, and normal findings as in Table 2 .

A positive moderate correlation (0.6) was found between lesions in CEDM and pathology. Most of 

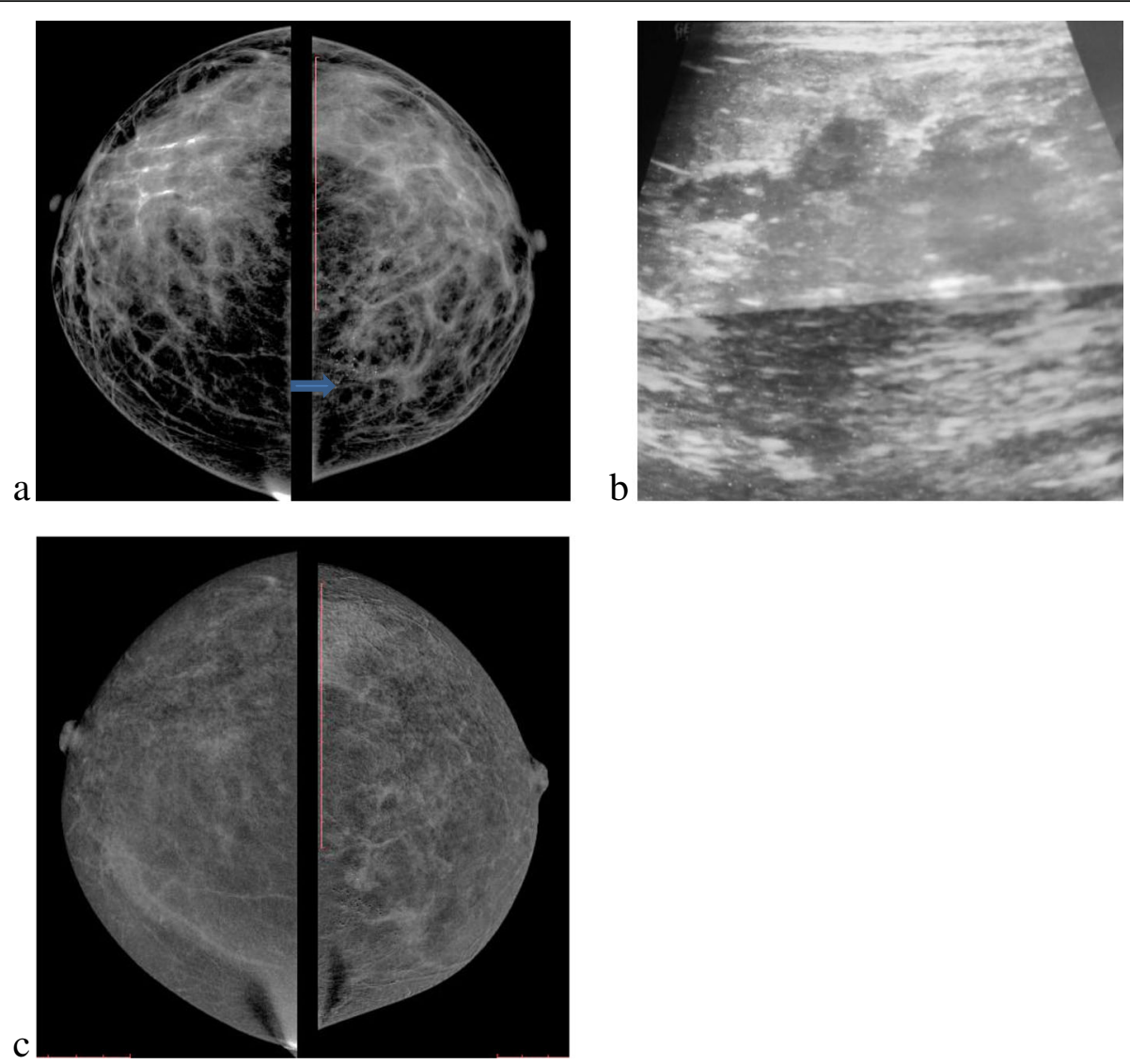

Fig. 4 A case of invasive duct carcinoma grade II showing in low energy image left focal asymmetry with suspicious calcifications (a). b Ultrasound shows an irregular mass lesion with superimposed microcalcifications. c Recombined image of CEDM shows two suspicious faintly enhancing mass lesions with irregular shape and speculated margins in the recombined image

malignant pathology was detected as mass enhancement while benign pathology and normality were proved in non-enhanced lesions. Most of lesions were diagnosed pathologically as IDC and showed moderate or intense enhancement.

\section{Pathological findings}

Most of the malignant findings were invasive ductal carcinoma (IDC), while abscess was the most common benign finding followed by fibroadenoma. Two cases were categorized as normal breast tissue as they did not show any enhancement or sonographic findings despite the presenting mammographic focal asymmetry, suggesting normal breast tissue.

CEDM and sonomammographic probabilities were compared to the pathological diagnosis as the reference standard in Table 2.

CEDM indices were compared to sonomammographic indices. CEDM had higher sensitivity, NPV, falsepositive results; however, sonomammography had higher specificity, PPV, and false-negative results (Table 3).
Compared multiplicity detection by sonomammography and CEDM revealed that more multiple lesions detection by CEDM (26.3\%) than sonomammography $(10.5 \%)$ with a moderate degree of accuracy in multiplicity detection is noticed in CEDM (0.49).

\section{Discussion}

The study aimed to evaluate diagnostic accuracy of CEDM for characterization of focal asymmetries in 2D mammography. Few studies evaluated this topic and most of them have investigated asymmetry in general $[3,7,8]$. However, this study focused exclusively on focal asymmetry. The overall results of our study confirmed the higher diagnostic accuracy of CEDM with second-look US than sonomammography only.

MRI lexicon for morphological analysis according to ACR 2018 classifies the lesions in subtracted images into focus, mass, and non-mass enhancement [12]. Our results showed a positive moderate correlation between lesions in CEDM and pathology (0.6) as majority of malignant pathology was detected as mass enhancement particularly and benign pathology and normality in non- 
a
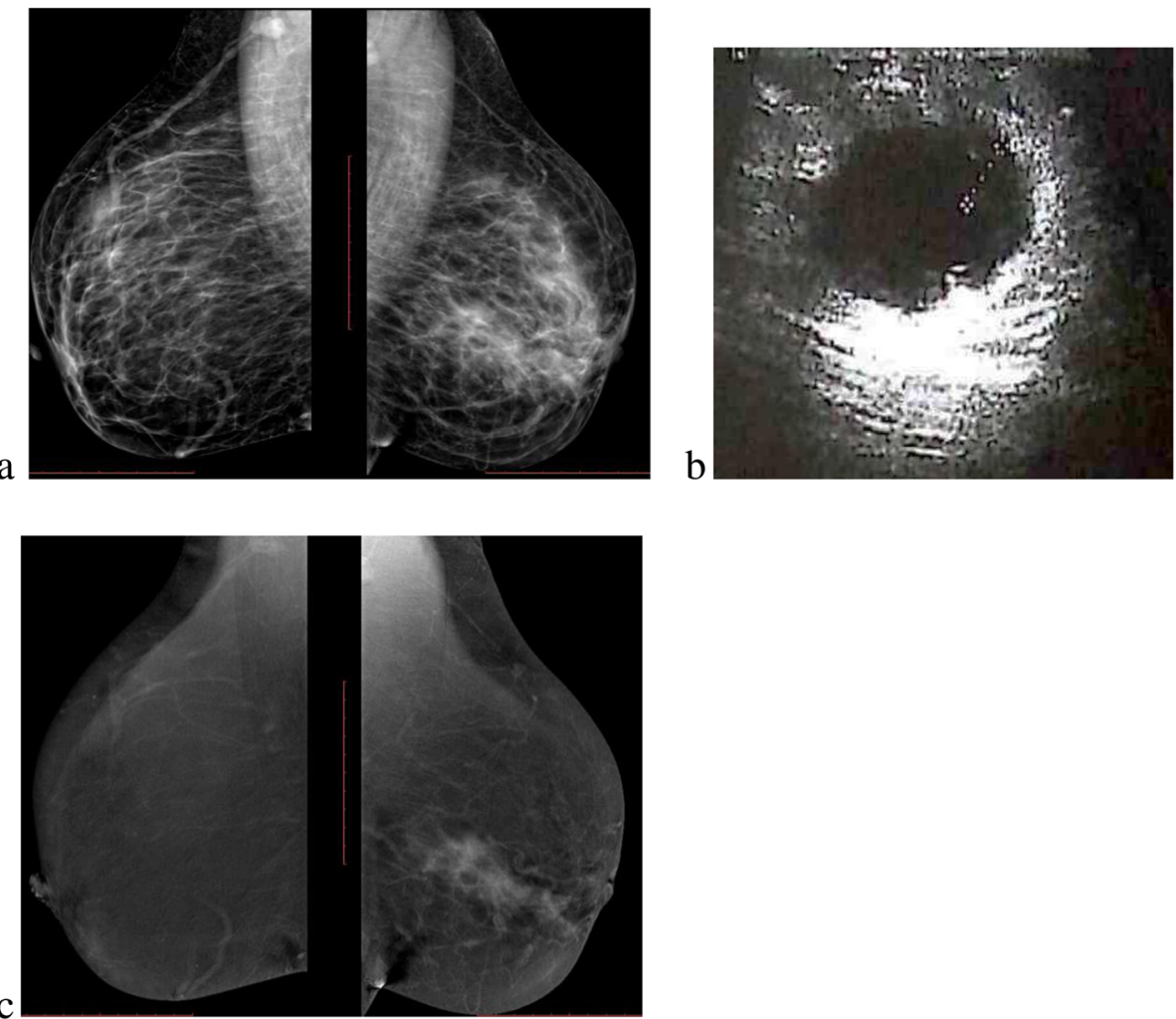

Fig. 5 A case of periductal mastitis with left focal asymmetry seen in digital mammography (a). b Ultrasound showed a focal area of increased echogenicity with a cystic lesion inside. c Recombined image of CEDM showing non-mass enhancement with associated rim enhancement

enhanced lesions. A recent study documented a significant correlation between asymmetry showing "mass or non-mass enhancement" and malignancy and between non-enhancing findings and benign pathology [8]. CEDM has the potential to depict angiogenesis, which is more pronounced in IDC $[13,14]$. As in our study, most of moderate or intense enhanced lesions were IDC.

As regard mass enhancement descriptors, the highest incidence of malignant lesions was correlated to irregular margin followed by irregular shape then intensity of enhancement and finally pattern of enhancement. A smooth circumscribed margin is seen in benign lesions versus a speculated or irregular margin seen in malignancy; heterogeneous enhancement pattern was exhibited mostly by malignant lesions. Dark septa are seen in fibroadenomas, which agree with other documentations $[12,15,16]$. Mass with rim enhancement is an unreliable sign in predicting malignancy. Ultrasound assessment of wall nodularity and thickness is vital $[12,15,16]$. In our finding about rim enhancement, one case was IDC and 4 cases were benign, namely, 3 abscesses cases and 1 granulomatous mastitis.

As regard non-mass enhancement descriptors in our study, suspicious heterogeneous pattern with segmental and regional distributions showed benign pathology and a single case of unilateral multiregional enhancement was malignant by pathology. In contrast to other literatures noticed the strong correlation between ductal/linear, segmental, focal distributions and heterogonous and clumped patterns with malignant pathology but regional, bilateral multiregional, and diffuse distribution were related to benignity $[6,12,17]$. Assessment of non-mass morphological descriptors is more subjective and gives more false-positive results [6].

Moreover, weak or absent enhancement characterizes benign lesions which agrees with our study as 4 cases of faint enhancement were benign lesions and all 5 nonenhancing lesions were benign lesions or representing normal parenchymal enhancement [13].

Invasive ductal carcinoma was described in a recent study as an intensely enhancing mass with speculated and irregular margins in majority of enhancing masses and much less presented as non-mass enhancement or non-enhancement and displayed no ring enhancement which partially agrees with our study as IDC presented as an intense or moderate enhancing mass with speculated and irregular margins in $95 \%$ of cases and as nonmass enhancement in $25 \%$ of cases, but rim enhancement was noticed in only one case of IDC [14].

The study showed that the case of faintly enhanced multiple foci was abscess which agrees with a recent 

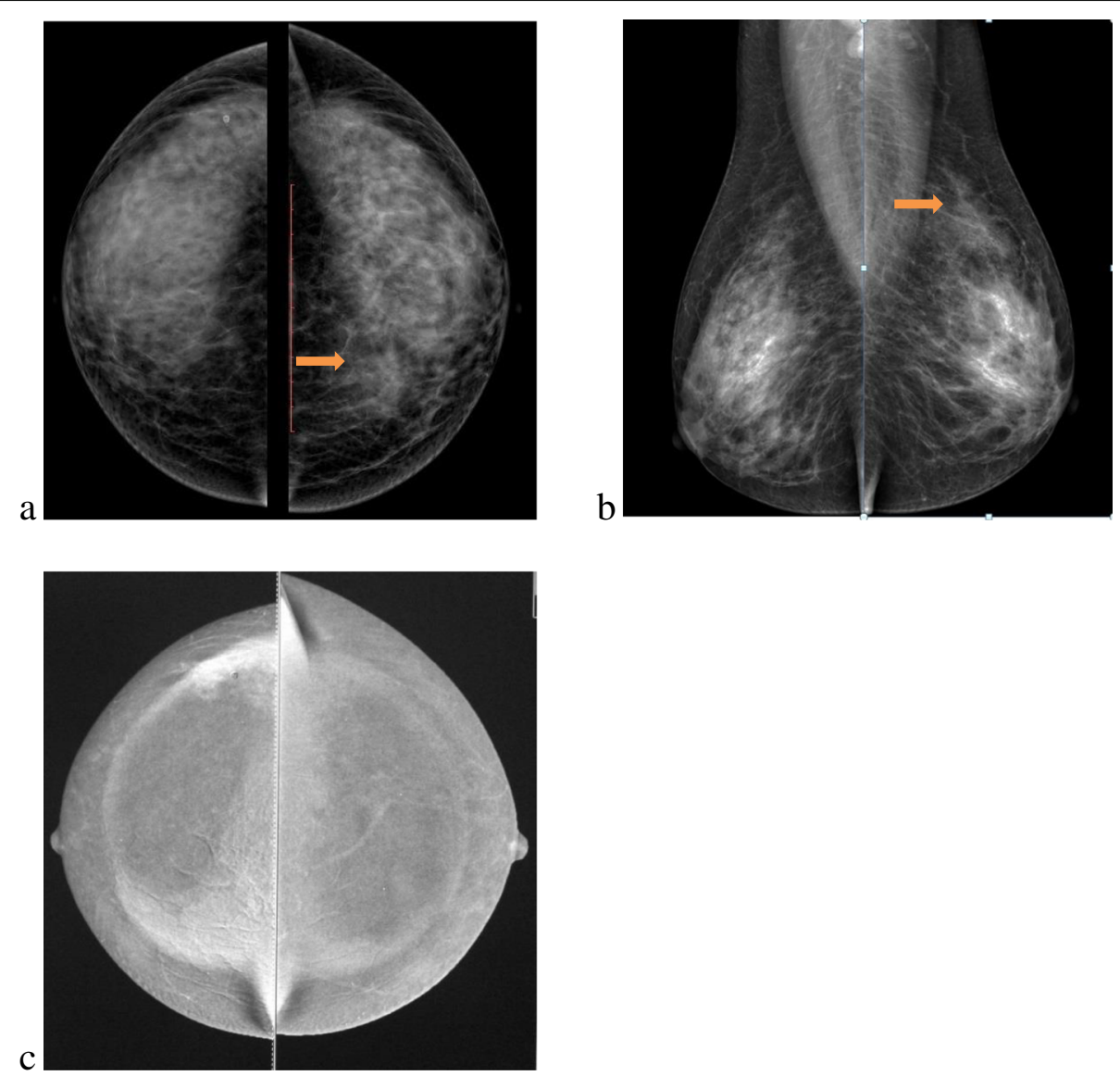

Fig. 6 A case presented with palpable lump at upper inner quadrant showing left focal asymmetry in CC (a) and MLO (b) views. US showed condensed glandular tissue. CEDM was requested by patient for reassurance and showed no enhancement in recombined image (c). Patient was reassured and recommended for routine follow-up

study revealed that the intense enhancement is the discriminating element in focus enhancement. Some studies reported that the faint bilateral foci could be considered as normal background parenchymal enhancement (BPE) after low energy images review and second look US $[6,7,16]$.

Microcalcifications with enhancement are favorable to malignant diagnosis, however, lack of enhancement may be diagnostic for non-malignant lesions or noninvasive

Table 3 Sonomammography vs. CEDM indices

\begin{tabular}{lll}
\hline & Sonomammography & CEDM \\
\hline Sensitivity & $65.7 \%(23 / 35)$ & $77.8 \%(21 / 27)$ \\
Specificity & $100.0 \%(3 / 3)$ & $81.8 \%(9 / 11)$ \\
PPV & $100.0 \%(23 / 23)$ & $91.3 \%(21 / 23)$ \\
NPV & $20 \%(3 / 15)$ & $60 \%(9 / 15)$ \\
False positive & $0 \%(0 / 3)$ & $18.2 \%(2 / 9)$ \\
False negative & $34.3 \%(12 / 35)$ & $22.2 \%(6 / 27)$ \\
Accuracy & 0.17 & 0.58 \\
\hline
\end{tabular}

subgroup cancers [4, 17]. Amorphous or pleomorphic calcifications $+/$ - mass on mammograms have high cancer prediction rate of DCIS lesions showing weak enhancement [13]. Previous studies are comparable to our study, which showed 12 cases of calcifications with mammographic focal asymmetry. Nine cases of suspicious calcifications showed intense and moderate enhancing mass (IDC).

When compared to sonomammography, CEDM showed higher sensitivity ( $77.8 \%$ vs. 65.7$)$, NPV (60\% vs. $20 \%$ ), and accuracy (0.6 vs. 0.2 ), yet lower specificity ( $81.8 \%$ vs. $100 \%)$ and PPV (91.3\% vs $100 \%)$. Our study agrees with another study comparing CEDM to digital mammography in terms of sensitivity ( $90.6 \%$ vs. $83.11 \%)$ and NPV (0.8 vs. 0.6), while disagrees in terms of specificity $(97.7 \%$ vs. $60.4 \%)$ and PPV (98.5 vs. 79$)$ (14). In our study, the higher sensitivity of CEDM reflected the higher value in diagnosis of true malignant cases, while higher specificity of sonomammography reflected higher ability in detection of true benign cases. However, specificity of CEDM in our study was less than 
sonomammography, but it is still acceptable and could be easily improved. PPV of sonomammography reflected the prominent malignant features by sonomammography more than CEDM and better chance for malignancy detection by combination of the 2 modalities. NPV of CEDM was much higher reflecting its higher value in diagnosis of negative or benign cases and avoidance of unnecessary biopsies.

No false-positive cases were detected by sonomammography but only 2 in CEDM (abscess and fibroadenoma), which could be improved by more working up on this new modality and more experience of readers. Falsenegative cases by CEDM were much less, reflecting overestimation of the focal asymmetry by sonomammography. CEDM has higher accuracy when compared to sonomammography ( 0.6 and 0.2 respectively) reflecting its higher reliability.

CEDM can detect multiple and bilateral lesions and it is recommended in high-risk patients $[18,19]$. The detection rate of multiplicity was higher in CEDM than sonomammography.

Some limitations were found in our study such as subjective assessment of the degree of enhancement on CEDM, the small number of patients in particular subgroups, as microcalcifications (8 cases) or in situ cancers ( 1 case), in addition to manual injection of contrast which hindered kinetic assessment of the lesions.

\section{Conclusion}

CEDM is more accurate and sensitive than 2D mammography in determination of normal tissue, benign, or malignant lesions and in detection of multiplicity in cases of mammographic focal asymmetry. A strong correlation was found between mass enhancement and malignancy as well as non-enhancement and benignity and normality. However, no correlation was found between non-mass enhancement and pathology. CEDM showed higher accuracy in detection of multiplicity. Undesired biopsies were avoidable with proper management of suspicious and malignant lesions.

\section{Abbreviations}

BI-RADS: Breast Imaging-Reporting and Data System; BPE: Background parenchymal enhancement; CC: Craniocaudal; CEDM: Contrast-enhanced digital mammography; DCIS: Ducal carcinoma in situ; EDH: Epithelial ductal hyperplasia; IDC: Invasive duct carcinoma; ILC: Invasive lobular carcinoma; LN: Lymph node; MLO: Mediolateral oblique; MRI: Magnetic resonant image; NPV: Negative predictive value; PPV: Positive predictive value; US: Ultrasound; GFR: Glomerular filtration rate

\section{Acknowledgements}

The authors thank Dr. Hanaa Abdelkader and Dr. Neiveen Shalaby professors of Radiodiagnosis, Faculty of Medicine, Ain Shams University, for valuable discussions regarding topics related to this manuscript.

\section{Authors' contributions}

ME contributed in surgical and clinical evaluation of the patient and writing the manuscript. SM contributed in statistical analysis and writing the manuscript. Nk analyzed and interpreted the radiological findings as well as contributed in writing the manuscript. GM contributed in radiological, statistical analysis, and writing the manuscript. The authors read and approved the final manuscript.

Funding

Not applicable.

\section{Availability of data and materials}

The data that support the findings of this study are available from [Radiodiagnosis Clinic Ain Shams University] but restrictions apply to the availability of these data, which were used under license for the current study, and so are not publicly available. Data are however available from the authors upon reasonable request and with permission of Radiodiagnosis Clinic Ain Shams University.

Ethics approval and consent to participate

The research has been done after approval of the Faculty of Medicine, Ain Shams University Research Ethics Committee (FMASU REC) FWA 000017585. FMASU MD 174/2017

The study is a prospective study. The procedure was explained for each patient and written informed consent was signed.

\section{Consent for publication}

Images in the manuscript are not personal.

\section{Competing interests}

The authors declare that they have no competing interests.

\section{Author details}

${ }^{1}$ Radiodiagnosis Department, Ain Shams University, Ramsees Street, Al Abasia, Cairo 11517, Egypt. ${ }^{2}$ General Surgery Department, Ain Shams University, Cairo, Egypt.

Received: 20 July 2020 Accepted: 10 November 2020

Published online: 02 December 2020

\section{References}

1. Dongola N (2016) Mammography in breast cancer Medscape. https:// emedicine.medscape.com/article/1948247-overview (Accessed 23 Dec 2018)

2. Zonderland H (2013) Smithuis R (2013) ACR BIRADS atlas: BI-RADS for mammography and ultrasound 2013 updated version http://www. radiologyassistant.nl/en/p53b4082c92130 (Accessed 8 Jan 2019)

3. Perry H, Phillips J, Dialani V et al (2019) Contrast-enhanced mammography: a systematic guide to interpretation and reporting. AJR 212:1-10 https:// www.ajronline.org/doi/abs/10.2214/AJR.17.19265

4. Lalji UC (2016) Introduction. In: Lalji UC (ed) Contrast-enhanced spectral mammography in clinical practice: results in a screening population, Maastricht, pp 7-12 https://cris.maastrichtuniversity.nl/files/7265116/c5322. pdf (Accessed 28 Mar 2019)

5. Tennant SL, James JJ, Cornford EJ et al (2016) Contrast-enhanced spectral mammography improves diagnostic accuracy in the symptomatic setting. Clin Radiol 71:1148-1155. https://doi.org/10.1016/j.card.2016.05.009

6. Bicchierai G, Naro FD, Amato F (2018) Cedm lexicon and imaging interpretation tips. In: Nori J, Kaur M (eds) Contrast-enhanced digital mammography (CEDM). Springer International Publishing AG, part of Springer Nature, pp 93-119. https://doi.org/10.1007/978-3-319-94553-8_9

7. Kamal RM, Helal MH, Wessam R (2015) Contrast-enhanced spectral mammography: impact of the qualitative morphology descriptors on the diagnosis of breast lesions. EJR 84:1049-1055. https://doi.org/10.1016/j.ejrad. 2015.03.005

8. Wessam R, Gomaa MMM, Fouad MA et al (2019) The added value of contrast-enhancing mammography in assessment of breast asymmetry. $\mathrm{Br} \mathrm{J}$ Radiol 92:20180245. https://doi.org/10.1259/bjr.20180245

9. O'Connor M (2019) American Society of Breast Surgeons release mammography screening guidelines based on individual risk. In: Healthcare Economics \& Policy https://www.breastcancer.org/research-news/asbrsissues-updated-screening-guidelines https://www.breastsurgeons.org/docs/ statements/Position-Statement-on-Screening-Mammography.pdf

10. Dromain C, Balleyguier C, Muller S et al (2006) Evaluation of tumor angiogenesis of breast carcinoma using contrast enhanced digital 
mammography. Am J Roentgenol 187:528-537. https://doi.org/10.2214/AJR. 05.1944

11. Kim EY, Youn I, Lee KH et al (2018) Diagnostic value of contrast-enhanced digital mammography versus contrast-enhanced magnetic resonance imaging for the preoperative evaluation of breast cancer. JBC 21:453-462. https://doi.org/10.4048/jbc.2018.21.e62

12. Kamal RM, Helal MH, Mansour SM et al (2016) Can we apply the MRI BIRADS lexicon morphology descriptors on contrast-enhanced spectral mammography? BJR 89:20160157https://pubmed.ncbi.nlm.nih.gov/273274 03/. https://doi.org/10.1259/bjr.20160157

13. Luczynska E, Niemiec J, Hendrick E et al (2016) Degree of enhancement on contrast enhanced spectral mammography (CESM) and lesion type on mammography (MG): comparison based on histological results. Med Sci Monit 22:3886-3893. https://doi.org/10.12659/MSM.9003710

14. Nori J, Bellini C, Piccolo C (2018) Malignant lesions. In: Nori J, Kaur M (eds) Contrast-enhanced digital mammography (CEDM). Springer, pp 169-184. https://doi.org/10.1007/978-3-319-94553-8

15. Tohamey YM, Youssry SW, Abd El Aziz Al (2018) Interpretation of patterns of enhancement on contrast-enhanced spectral mammography: an approach to a standardized scheme. Egyptian J Radiol Nuclear Med 49:854-868. https://doi.org/10.1016/j.ejrnm.2018.03.004

16. El-Sharawy AA, Helal MA, Selim AS, Wessam R, El-Azab M, Gouda I (2017) The additional role of dual-energy contrast-enhanced digital mammography in characterization of breast lesions. MJCU 85:583-591 medicaljournalofcairouniversity.net/home2/images/pdf/2017/../a20.pd.www. medicaljournalofcairouniversity.net

17. Cheung YC, Juan YH, Lin YC et al (2016) Dual-energy contrast-enhanced spectral mammography: enhancement analysis on BI-RADS 4 non-mass microcalcifications in screened women. PLoS One 11(9). https://doi.org/10. 1371/journal.pone. 0162740

18. Jochelson MS, Dershaw DD, Sung JS (2013) Bilateral contrast-enhanced dual-energy digital mammography: feasibility and comparison with conventional digital mammography and MR imaging in women with known breast carcinoma. Radiology 266:743-751. https://doi.org/10.1148/ radiol.12121084

19. Aja MMT, Rodríguez MR, Hernández SA, Benítez W, Luzardo OP (2014) Dualenergy contrast-enhanced mammography. Radiología 56:390-399. https:// doi.org/10.1016/j.rx.2014.05.003

\section{Publisher's Note}

Springer Nature remains neutral with regard to jurisdictional claims in published maps and institutional affiliations.

\section{Submit your manuscript to a SpringerOpen ${ }^{\circ}$ journal and benefit from:}

- Convenient online submission

- Rigorous peer review

- Open access: articles freely available online

- High visibility within the field

- Retaining the copyright to your article

Submit your next manuscript at $\boldsymbol{\nabla}$ springeropen.com 